\title{
Study of brain tissue impedance in the hydrocephalic cat
}

\author{
KENICHIRO HIGASHI, * YASUTAKA NODA, $\dagger$ SHUNRO TACHIBANA
}

From the Neurosurgical Research Institute, Kitakyushu Central Hospital, Kitakyushu, ${ }^{*}$ Institute of Animal Experiment, Kurume University Medical School, Kurume, $\dagger$ and Wakasugi Medical Research Institute, Sasaguri, Fukuoka $\ddagger$ Japan

SUMMARY Impedographic studies were made in cats made hydrocephalic by intracisternal kaolin. Enlargement of the anterior horns preceded that of the body of the lateral ventricles, and there was a selectivity to pressure atrophy among different tissues within the brain. Furthermore, sequential measurement of impedance using chronically implanted electrodes demonstrated a gradual decrease of impedance in the periventricular white matter during the development of hydrocephalus, suggesting an expansion of the extracellular space in the tissue around the ventricular wall.

It is well known that the principal pathogenic mechanism of hydrocephalus is disturbance in cerebrospinal fluid circulation. However, there is limited knowledge of the pathophysiological processes within the brain tissues.

Impedography, developed by one of the authors (ST) in 1970," "scans" the interior of the brain by recording tissue impedance around the tip of an electrode moved through multiple, closely spaced, parallel trajectories. By employing analogue display of these data, we can reconstruct the gross morphology of a brain along any selected plane. We employed this technique to investigate the morphological and pathophysiological changes in hydrocephalic cats. Furthermore, sequential measurements of cerebral tissue impendance were carried out, using chronically implanted electrodes, in order to investigate the electrochemical changes in the hydrocephalic brain.

\section{Method}

Technique for recording cerebral impedography

Forty nine adult cats weighing 2.8 to $4.0 \mathrm{~kg}$ were used in the experiments. Animals were anaesthetised with intraperitoneal pentobarbital sodium $(30 \mathrm{mg} / \mathrm{kg})$ and placed in a stereotaxic head holder. After making a midline scalp incision, a narrow transverse window was cut in the skull ( $1.5 \mathrm{~mm}$ wide) parallel to the coronal suture using a dental drill. Care was taken to avoid injuring the dura mater at the

Address for reprint requests: Kenichiro Higashi, MD, Department of Neurosurgery, Kitakyushu Central Hospital, 1-4-5 Shiragane, Kokurakita-ku, Kitakyushu, 802, Japan.

Received 4 March 1988 and in revised form 5 July 1988. Accepted 5 November 1988 time of making the skull window. A shallow well, formed by elevating the skin edges with a ligature, was filled with saline. Each electrode was positioned initially in the saline and then driven through the brain from this narrow window without removing the dura mater. An indifferent electrode, consisting of a stainless steel disc, $1 \mathrm{~cm}$ in diameter, was placed subcutaneously over the frontal bone. The tissue impedance was measured at multiple points in the brain and recordedO sequentially by repeated insertion, withdrawal and horizontal movement of the electrode using a microdrive system which consisted of a probe drive with micrometer and a variable-speed motor. Insertion of the electrode started at the left lateral edge of the skull window (approximately $2 \mathrm{~cm}$ from the midline) and the lowest point of electrode travel was usually $1 \mathrm{~mm}$ above the floor of the skull, so as to protect the electrode tip from damage. Thus the depth of the electrode insertion ranged from 25 to $35 \mathrm{~mm}$ from the dural surface. Sequential parallel insertions were made at $0.5 \mathrm{~mm}$ intervals, and the rate of electrode movement was $1 \mathrm{~mm} / \mathrm{s}^{1}$ The semimicro electrode for recording of impedance consists of a $0.1 \mathrm{~mm}$ diameter, $30 \mathrm{~mm}$ long rhodium needle insulated with thin epoxy resin except for $0.2 \mathrm{~mm}$ at the tip. The impedance recording system consists of an ohmmeter circuit driven with a high frequency sine wave. To take a picture of a given cerebral plane, impedance is monitored continuously from serial parallel electrode trajectories through the brain. The impedance signal provides the input for intensity modulation (Z-axis) of an oscilloscope, and the electrode location (depth) is indicated by $\mathbf{Y}$-axis with adjustment of beam sweep and electrode speed. Sequential profiles obtained from parallel tracks are plotted on the $\mathrm{X}$-axis and the resulting image is photographed with a polaroid camera. The method of the impedography has been described in detail in the previous report by one of the authors.'

Method for production of experimental hydrocephalus

The cisterna magna of anaesthetised cat was punctured by a 23 gauge needle. After removal of $0.8 \mathrm{ml}$ of CSF, the same 
amount of $250 \mathrm{mg} / \mathrm{ml}$ suspension of sterilised kaolin in saline was injected into the cisterna magna. Animals became inert and did not take food by themselves for 2-3 days. Therefore, forced feeding or infusion of nutrient was usually needed.

Performance of cerebral impedography

Fifty seven impedographies of living brain were carried out at various periods from 1 to 9 weeks after intracisternal kaolin injection in 32 cats. Impedography of normal living brain was also done in 9 untreated cats. After completion of the impedography, the brain was perfused with saline followed by $10 \%$ formalin through the catheters inserted into the bilateral common carotid arteries. The brain was removed and immersed in $10 \%$ formaline solution thereafter. Impedography of the dead brain was performed on the formalin-fixed brain in 27 cats after immersion in saline for 24 hours and then embedded with gelatine.

In 12 animals, impedography of the normal brain was carried out 2-3 weeks prior to intracisternal injection of kaolin, then repeated 2 weeks thereafter. In this manner, observations of the interior of the brain were available in the same animal during the progression of the hydrocephalus. Impedography of the living brain was done at the desired coronal plane level. In the dead brain, however, several different planes were chosen.

Sequential measurements of tissue impedance of the hydrocephalic brain by chronically implanted electrodes The bipolar impedance electrodes were implanted into the periventricular white matter, corpus callosum, caudate nucleus, thalamus and midbrain reticular formation in two cats. The electrode consisted of two parallel $32 \mathrm{G}$ nichrome wires $1 \mathrm{~mm}$ apart, insulated except at the cut ends with epoxy resin. Two months later, when the excitability of these brain tissues due to electrode insertion was reduced, and the stability of the electrode implants was secured, kaolin suspension was injected into the cisterna magna. Tissue impedance was then measured repeatedly by each electrode before and every 2 to 3 weeks after kaolin injection.

Measurements of impedance were made with a sine-wave signal at a frequency of $20 \mathrm{kHz}$. The bipolar electrode formed one arm of a Wheatstone bridge. During impedance measurements, $2 \mu \mathrm{a}$ of high frequency current passed through the brain, and resistance and capacitance of the bridge were completely balanced at each measurement using a cathode ray oscillograph. The measurement procedures have been described in detail in the previous reports. ${ }^{23}$

\section{Results}

Relation between time-course after intracisternal kaolin injection and progression of ventricular dilatation

The grade of ventricular dilatation was evaluated by
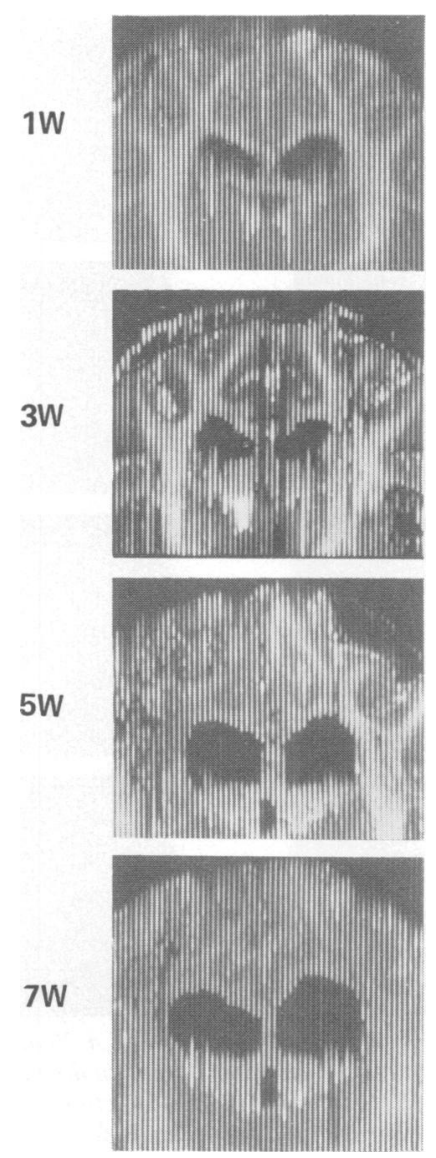

Fig 2 Sequential impedographs of the same living cat brain at 2 weeks intervals along with development of hydrocephalus. Pictures are arranged from the top, impedographs taken at 1 , 3,5 and 7 weeks following intracisternal injection of kaolin. Coronal section, $A-12$.

both impedographic images and in brain slices. Excluding 8 animals which died within a week following kaolin injection, ventricular dilatation was observed in 30 of 32 cats $(93.8 \%)$.

Impedographs show striking similarities to the cut surface of the brain. White matter has high impedance which appears light, and gray matter has low impedance shown as darker tones. Cerebrospinal fluid has the lowest impedance, so that the ventricles are
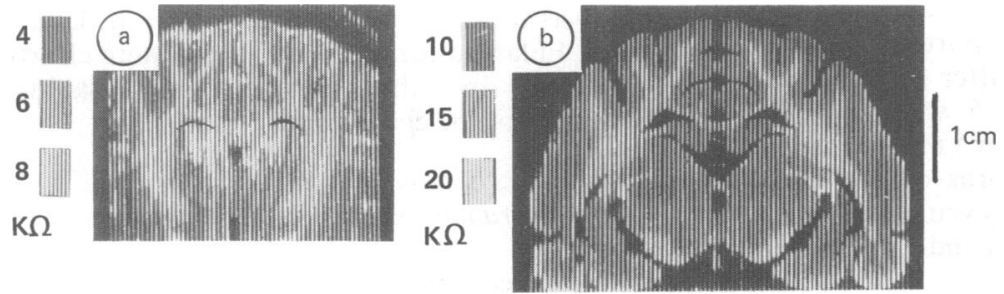

Fig 1 Impedographs of coronal section of the normal cat brain (level A-12).

A: Living brain. B: Formalin fixed dead brain. Intensity modulated impedance calibration is shown for equivalent resistance. 

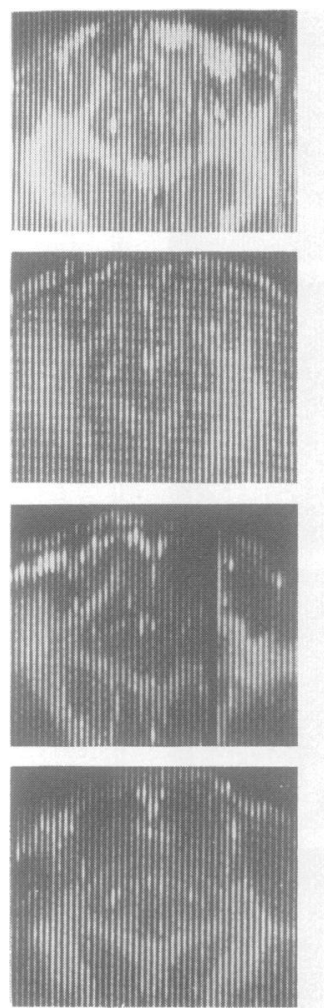
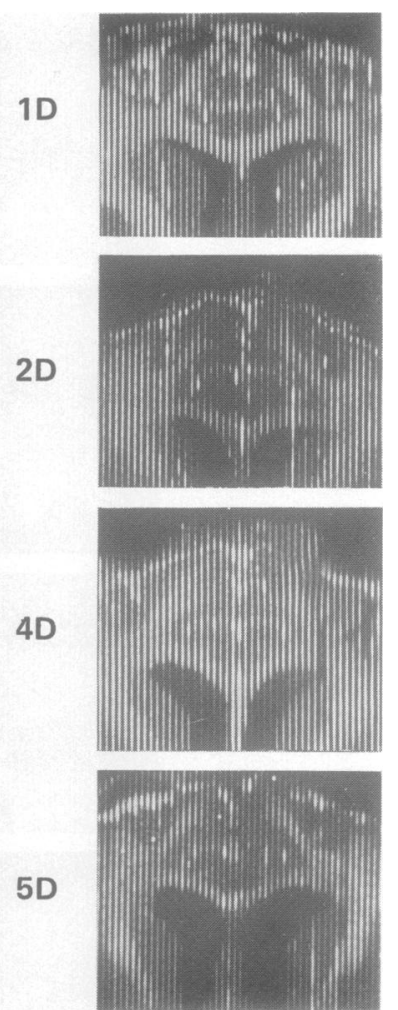

Fig 3 Impedographs of coronal section, A-20 of the living cat brain before and after intracisternal kaolin injection. Pictures of the left-hand side are pre-injection control impedographs. Those of the right-hand side are impedographs taken at 1, 2, 4 and 5 days following kaolin injection. Experiments were carried out in four different cats. Each control impedograph corresponds to post-injection impedograph of just right-hand side.

sharply delineated in both size and shape. The lateral ventricles of normal cat appears as slits or narrow gaps as demonstrated in fig 1 .

Since impedography with $0.1 \mathrm{~mm}$ electrodes is a relatively harmless procedure, we can carry out several examinations in the same living animal. Figure 2 shows sequential impedographs at 2 week intervals during the development of hydrocephalus following intracisternal kaolin injection. The gradual progress of ventricular enlargement is well documented. In this level of the coronal plane A-12, that is, $12 \mathrm{~mm}$ anterior to the centre of the external acoustic porus, ventricular dilatation was not remarkable after 1 week, but became conspicuous after 3 to 5 weeks. In the impedographs at the level of A-17, or $5 \mathrm{~mm}$ anterior to the previous one, the anterior horns of the lateral ventricles were dilated remarkably even after 1 week, and they continued to enlarge slowly and progressively therafter.
Higashi, Noda, Tachibana

We also tried to make impedographs at a more anterior plane and at an earlier stage than 1 week. Figure 3 shows the impedographs at the level of A-20, or $8 \mathrm{~mm}$ anterior to A-12. With these figures, it is demonstrated that in the most anterior part of the lateral ventricles, enlargement occurred as early as 24 hours after intracisternal kaolin injection. It rapidly progressed and was very pronounced after 5 days. Thus we observed a difference in the development of ventricular dilatation depending on the location of the ventricles. As the ventricular dilatation progressed, atrophy of the periventricular white matter and the caudate nucleus became evident. The periventricular lucency which is usually seen in the computerised tomography (CT) of developing hydrocephalus was not observed in the impedographs.

On the impedographs at the above mentioned three levels of the coronal plane, we measured the width of the caudate nucleus, corpus callosum, periventricular white matter, and thalamus and compared these with results in control animals. Measurements were also made in the course of progression of hydrocephalus. The changes of the tissue width are demonstrated in fig 4. The sites of measurement for each tissue are shown in the diagram on the left hand side of the figure. In the periventricular white matter and the caudate nucleus, progressive atrophy occurred during the development of hydrocephalus. Also there was a smaller reduction in thalamic width. On the other hand, the width of the corpus callosum did not change significantly.

Comparison between impedographs of living and dead brains

In general, tissue impedance was higher in the dead brain than in the living brain; impedance value ranged from 10 to $20 \mathrm{kohms}$ in the dead brain, while that was 3 to $10 \mathrm{kohms}$ in the living brain. Therefore, contrast of the picture was sharper in the impedograph of the dead brain. (fig 1). However, there was no difference between the gross impedographic images of living and dead brains. In the case shown in fig 5 , following performance of impedography of living brain at the seventh week after intracisternal kaolin injection, impedographs were made of the dead brain at the coronal and sagittal planes. The latter was performed at the level of $5 \mathrm{~mm}$ lateral to the midline. These pictures were compared with the brain slice of the coronal plane. In this case, enlargement of the third ventricle was conspicuous as well as that of the lateral ventricles. Enlarged foramina of Monro were clearly demonstrated in the coronal and sagittal impedographs of the dead brain.

Serial recordings of tissue impedance of the hydrocephalic brain using chronically implanted electrodes

Sequential direct measurement of impedance in 


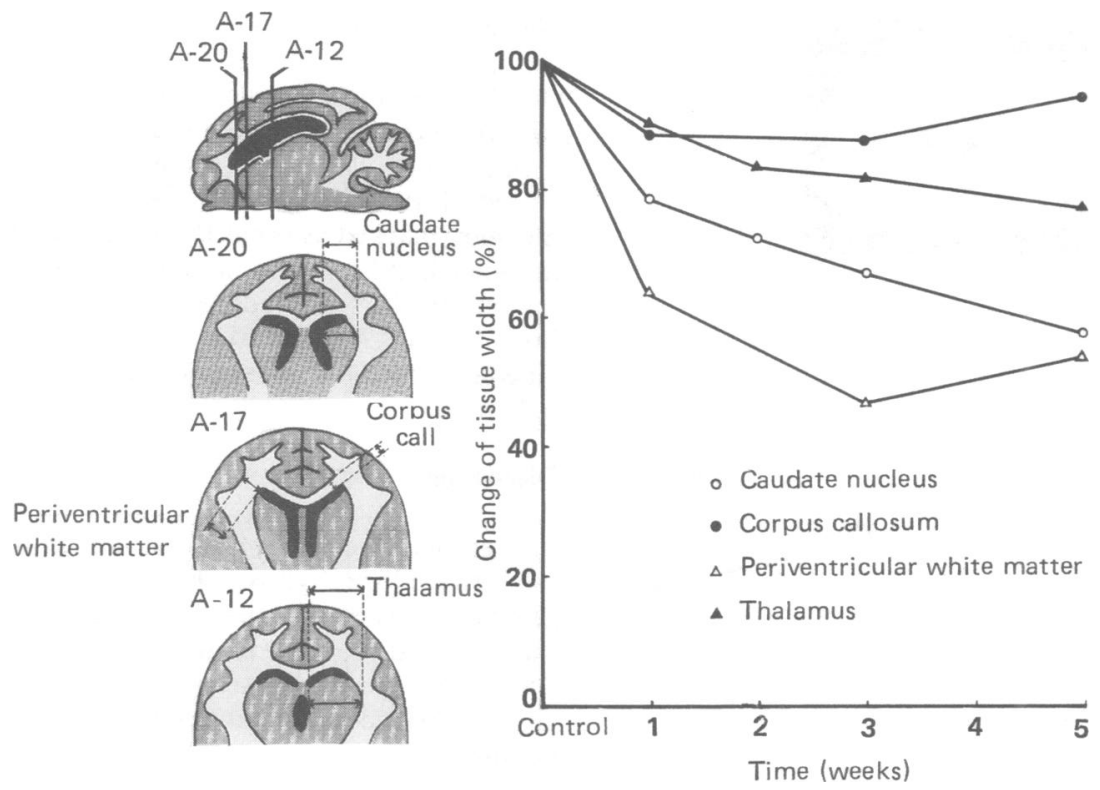

Fig 4 Changes in width of various tissues of the cat brain along with the progression of hydrocephalus, measured on sequential impedographs recorded at 1 to 5 weeks following intracisternal kaolin injection. The site of measurement for each tissue are shown in diagrams.

several tissues of hydrocephalic brain was carried out by chronically implanted electrodes in two cats. The target points include the periventricular white matter, corpus callosum, caudate nucleus, thalamus and midbrain reticular formation.

Figure 6 shows changes of tissue impedance in the
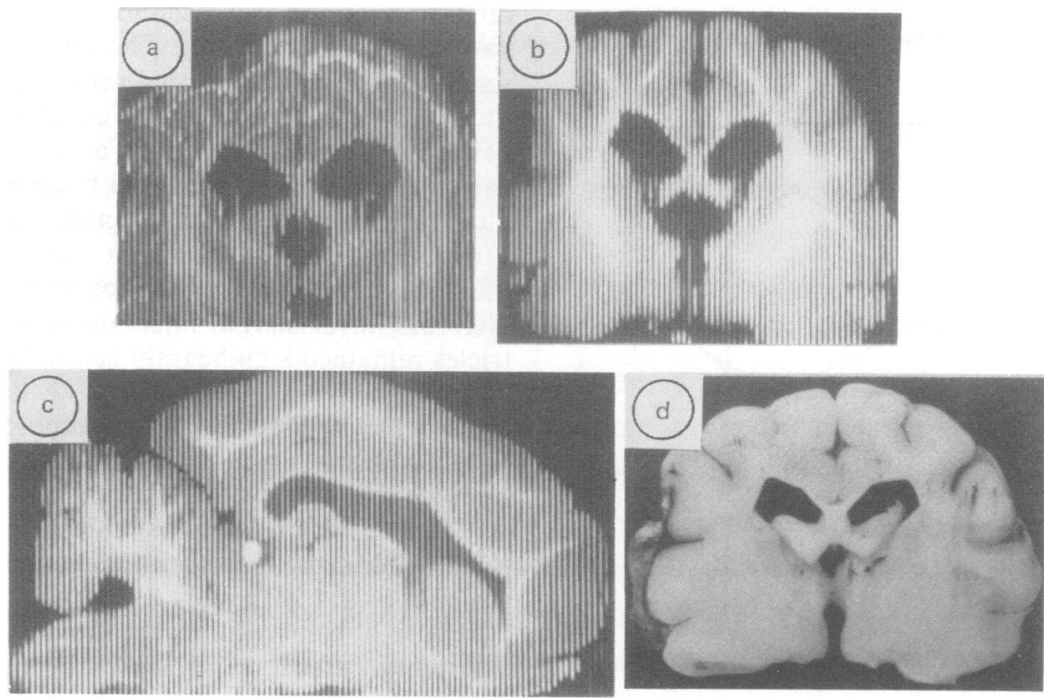

Fig 5 Comparison of impedographs of the living and dead brain and the brain slice in the hydrocephalic cat 7 weeks after intracisternal injection of kaolin. a: Impedograph of coronal section, A-12, of the living brain. $b$ : Impedograph of the same level of the formalin-fixed dead brain of the same cat. $c$ : Impedograph of sagittal section of the dead brain; $5 \mathrm{~mm}$ lateral to the midbrain. $d$ : Coronal section of the formalin-fixed brain corresponding to the level of the impedographs $A$ and $B$. 

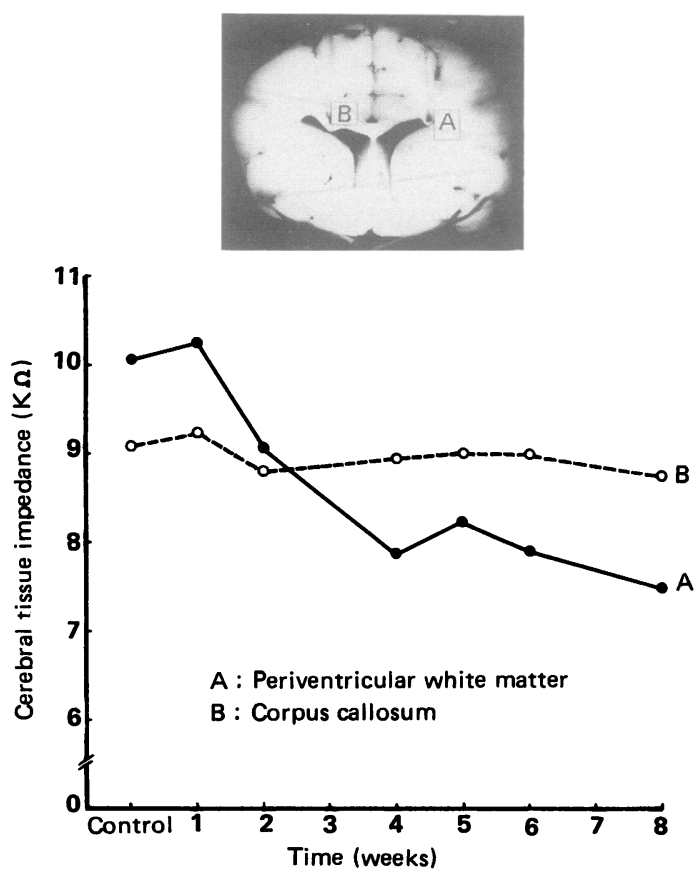

Fig 6 Changes of tissue impedance in the subependymal tissues around the anterior horn of the lateral ventricle along with developmental course of hydrocephalus following intracisternal kaolin injection, the tracks of the electrodes are shown in the coronal section of the brain; the tips are located in periventricular white matter around the lateral angle of the anterior horn $(A)$ and the corpus callosum $(B)$.

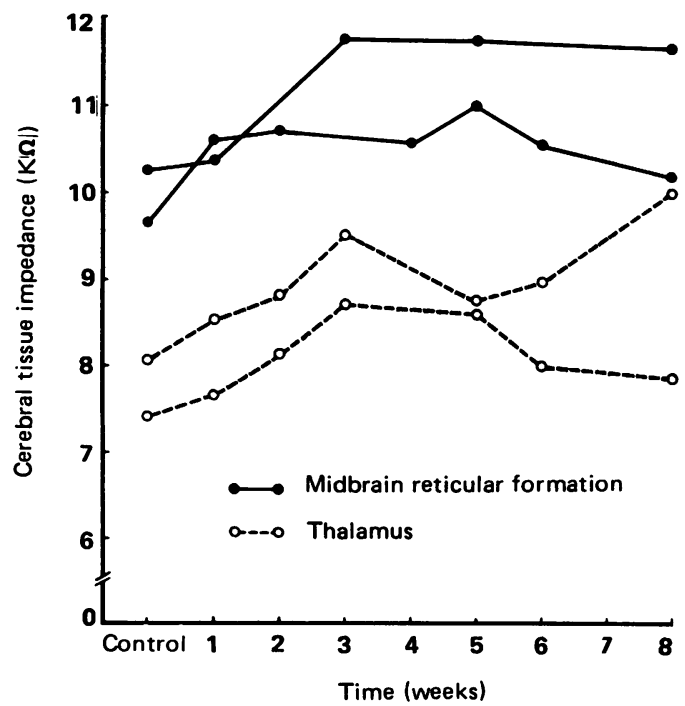

Fig 7 Changes of tissue impedance in the midbrain reticular formation and the thalamus along with development of hydrocephalus. subependymal tissues around the anterior horns of the lateral ventricles. In the white matter adjacent to the lateral angle of the anterior horn, which is marked as point $A$ on the section of the brain, a gradual decrease of impedance was observed as hydrocephalus progressed. Little change was observed in the corpus callosum, marked as point $B$ on the brain section. On the other hand, a $15 \%$ increase of tissue impedance was observed in the midbrain reticular formation as shown in fig 7. Similarly, a temporal increase of impedance of $20 \%$ was seen in the central part of the thalamus.

\section{Discussion}

Intracranial injection of kaolin suspension is widely used to induce hydrocephalus in animals. ${ }^{45}$ It is generally accepted that hydrocephalus in this experimental model caused by an impairment of the CSF circulation and absorption due to aseptic meningitis or obstruction of subarachnoid CSF pathway by kaolin. ${ }^{6}$

Although brain morphology can be observed serially during development of hydrocephalus, only a few reports have been published about the changes in the size of the ventricular system in kaolin-treated animals. Granholm ${ }^{7}$ studied the size of the ventricles by means of planimetry of the ventricular surface in coronal section of rabbit's brain which had been treated by intracisternal kaolin. He found that the dilatation of the ventricles occurred soon after the kaolin injection, and they became more than four times larger than the normal ventricles at 7 days after the injection. Edvinsson and West ${ }^{8}$ studied the relation between intracranial pressure and ventricular size at various stages of experimental hydrocephalus in rabbit. They observed a highly significant increase in the ventricular size concomitant with a remarkable increase in ventricular fluid pressure (VFP) 2 days after the intracisternal injection of kaolin. The ventricles remained significantly larger than those in the controls 7 and 30 days after the kaolin injection, though the VFP had returned almost to the control level. Murata et al $^{9}$ studied ventricular size in hydrocephalic dog using CT images and stated that the degree of ventricular enlargement was most remarkable in the first week and reached the maximum size in the 4th to 6th week.

By use of cerebral impedography, we can visualise the interior structure of the brain in living animals. The appearance is similar to a cut surface of the brain. Since the impedograph is constructed from the integrated signals of numerous sequential electrode insertions, a minimum of tissue injury is unavoidable, even with use of a fine electrode. However, such an injury is not serious enough to interfere with the 
reconstruction of a precise impedographic replica of the actual gross structure in the same plane; thus it allows repeated impedographic observations of the brain in the same animal along with developmental process of hydrocephalus.

Following intracisternal injection of kaolin suspension in cats, impedographic follow-up along with the developing process of hydrocephalus revealed that there was a certain pattern of progression of ventricular enlargement. The most anterior portion of the lateral ventricles enlarged from a very early stage even within 24 hours after kaolin injection, while in the body of the lateral ventricles, progression of enlargement became remarkable at the stage of 3 to 5 weeks and reached the maximal dilatation at 5 to 7 weeks after the kaolin injection. The impedographic image demonstrated selectivity to pressure atrophy among different tissues within the brain; in other words, there is a difference in vulnerability of tissue to increased VFP. It is interesting to note that, even in the tissues adjacent to the ventricular walls, the corpus callosum appeared to be more resistant to increased VFP than were periventricular white matter and the caudate nucleus.

Because impedance represents resistance to electric current conduction in the tissue, brain tissue impedance has been used neurosurgically to determine either brain tumour localisation ${ }^{10}$ or localisation of a target during stereotaxic surgery. "At the same time, basic research in this field has demonstrated an intimate relationship among tissue impedance, anatomical structures, and neuronal activity under physiologial and pathological states. ${ }^{12-16}$ According to these studies, the major determinant of impedance within cerebral tissue is assumed to be the extracellular space size and the character of its electrolyte content. Van Harreveld ${ }^{1718}$ stated that when intracellular swelling took place, shift of fluid from extracellular to intracellular space lead to increase in tissue impedance due to gradual occlusion of the extracellular space. Such intracellular swelling might be seen as a white image in an impedograph. Conversely, enlargement of extracellular space brought about decrease in tissue impedance, is observed as dark tone on the impedograph. Experimentally, an increase of impedance has been observed in anoxia which, in some instances, may be accompanied by cytotoxic brain oedema. ${ }^{1819}$ Conversely, a decrease of impedance occurs in vasogenic brain oedema by cold induction ${ }^{20}$ or caused by penetration injury of the brain. ${ }^{21}$

In CT of the hydrocephalic brain, periventricular lucency is usually observed during the acute stage. This hypodensity of CT image is thought to represent an oedematous change of the periventricular white matter, resulting from forced movement of ventricular fluid into the tissue as a compensated route of absorption of CSF. ${ }^{22}$ In the cerebral impedograph, we failed to find any low impedance zone in the tissue adjacent to the ventricles in hydrocephalic brain. The reason for this discrepancy between the two kinds of images is unknown. The absence of a periventricular low impedance area may be due either to the resolution of the impedograph being insufficient to depict such a subtle change, or to the different mechanisms contributing to impedographic imaging. However, study with chronically implanted electrodes revealed that there was a gradual decrease of impedance in the periventricular white matter along with the development of hydrocephalus. This may be evidence of expansion of extracellular space in restricted sites of periventricular tissue of hydrocephalic brain. On the other hand, an increase of impedance was observed during development of hydrocephalus in some tissues relatively remote from the wall of the ventricles such as the midbrain reticular formation and central part of the thalamus. This increased impedance is supposed to result from shrinkage of extracellular space due to compression by elevated ventricular pressure.

Observation of impedance in the hydrocephalic brain allowed definition of changes in both the morphology and physical characteristics of the brain secondary to hydrocephalus. However, the significance of these changes in brain impedance is not fully understood and may relate to changes in neuronal function that occur in many pathological conditions. Further studies should be undertaken to relate the change in impedance with specific electrochemical properties in the hydrocephalic brain which may lead to an understanding of disturbed function that may occur with this condition.

\section{References}

1 Tachibana S, Aguilar JA, Birzis L. Scanning the interior of living brain by impedography. J Appl Physiol 1970;28:534-9.

2 Birzis L, Tachibana S. Measurement of local cerebral blood flow by impedance changes. Life Sci 1962;11:587-98.

3 Birzis L. Impedance as a measure of flow velocity in salt solutions. J Appl Physiol 1966;21:329-33.

4 Bering EA Jr, Sato O. Hydrocephalus: Changes in formation and absorption of cerebrospinal fluid within the cerebral ventricles. $J$ Neurosurg 1963;20:1050-63.

5 Hochwald GM, Sahar A, Sadik AR, Ransohoff J. Cerebrospinal fluid production and histological observations in animals with experimental obstructive hydrocephalus. Exp Neurol 1969; 25:190-9.

6 Schurr PH, McLaurin RL, Ingraham FD. Experimental studies on the circulation of the cerebrospinal fluid and methods of producing communicating hydrocephalus in the dog. $J$ Neurosurg 1953;10:515-25.

7 Granholm L. Induced reversibility of ventricular dilatation in experimental hydrocephalus. Acta Neurol Scand 1966;42:581-8.

8 Edvinsson L, West KA. Relation between intracranial pressure and ventricular size at various stages on experimental hydrocephalus. Acta Neurol Scand 1971;47:451-7. 
9 Murata T, Mori K, Handa H, Nakano Y. Computed tomography on experimental canine hydrocephalus. Part 1. Observations on changes of ventricular size and periventricular lucency. Brain Nerve (Tokyo) 1978;30:677-85 (Jpn).

10 Organ LW, Tasker RR, Moody NR. Brain tumor localization using an electrical impedance technique. $J$ Neurosurg 1968;28:35-44.

11 Laitinen LV, Johansson GG, Sipponen P. Impedance and phase angle as a location method in human stereotaxic surgery. $J$ Neurosurg 1966;25:628-33.

12 Aladjalova NA. Slow electrical process in the brain. Prog Brain Res 1964;7:156-206.

$13 \mathrm{Li} \mathrm{CL}$, Bak AF, Parker OR. Specific resistivity of the cerebral cortex and white matter. Exp Neurol 1968;20:544-57.

14 Ranck JB. Analysis of specific impedance of rabbit cerebral cortex. Exp Neurol 1963;7:144-52.

15 Tachibana S. Homeostatic mechanism in the hypothalamus. Brain Res 1969;13:522-40.
16 Tarkkanen L. Thalamic impedance in man. Ann Clin Res 1970;2(suppl 4):1-63.

17 Van Harreveld A, Crowell J, Malhotra SK. A study of extracellular space in central nervous tissue by freeze substitution. J Cell Biol 1965;25:117-37.

18 Van Harreveld A, Ochs S. Cerebral impedance changes after circulatory arrest. Am J Physiol 1965;187:180-92.

19 Gazendam J, Go KG, Van der Meer JJ, Zuiderveen F. Changes of electrical impedance in edematous cat brain during hypoxia and after intracerebral ouabain injection. Exp Neurol 1979;66: 78-87.

20 Van der Veen PH, Go KG, Zuiderveen F, Buiter D, Van der Meer J. Electrical impedance of cat brain with cold-induced edema. Exp Neurol 1973;40:675-82.

21 Tachibana S. Impedance study of brain tissue changes after penetrating injury. Exp Neurol 1971;32:206-17.

22 Mori K, Murata T, Nakano Y, Handa H. Periventricular lucency on CT in hydrocephalus. Surg Neurol 1977;8:337-40. 